\title{
Connection problem for the tau-function of the Sine-Gordon reduction of Painlevé-III equation via the Riemann-Hilbert approach.
}

\author{
A. Its \\ Department of Mathematical Sciences, \\ Indiana University - Purdue University Indianapolis \\ Indianapolis, IN 46202-3216, USA \\ A. Prokhorov \\ Department of Mathematical Sciences, \\ Indiana University - Purdue University Indianapolis \\ Indianapolis, IN 46202-3216, USA
}

\begin{abstract}
We evaluate explicitly, in terms of the Cauchy data, the constant pre-factor in the large $x$ asymptotics of the Painlevé III tau-function. Our result proves the conjectural formula for this pre-factor obtained recently by O. Lisovyy, Y. Tykhyy, and the first co-author with the help of the recently discovered connection of the Painlevé tau-functions with the Virasoro conformal blocks. Our approach does not use this connection, and it is based on the RiemannHilbert method.
\end{abstract}

\section{Introduction}

We consider the particular case of the third Painlevé equation, which is a radial-symmetric reduction of the elliptic sine-Gordon equation

$$
u_{x x}+\frac{u_{x}}{x}+\sin u=0
$$

Starting from the pioneering works [1], [15] on the Ising model, this equation has been playing an increasingly important role, as a "nonlinear Bessel function", in a growing number of physical applications (see e.g., [4] and references therein). Apparently, the first appearance of equation (1) in the physical applications should be credited to work of J. M. Myers [16].

Equation (1) can be written as a (non-autonomous) Hamiltonian system,

$$
\frac{d u}{d x}=\frac{\partial \mathcal{H}}{\partial v}, \quad \frac{d v}{d x}=-\frac{\partial \mathcal{H}}{\partial u},
$$


on the phase space $\mathbb{R}^{2}=\{(u, v)\}$ equipped with the canonical symplectic structure,

$$
\Omega=d v \wedge d u \text {. }
$$

The Hamiltonian $\mathcal{H}$ is given by the formula

$$
\mathcal{H}=\frac{v^{2}}{2 x}-x \cos u
$$

We are concerned with the global asymptotic analysis of the $\tau$-function corresponding to the Painlevé III equation (1) which is defined according to the equation (see [9], [19]),

$$
\frac{d \ln \tau}{d x}=-\frac{1}{4} \mathcal{H}
$$

In fact, it is this tau-function (evaluated for a special family of solutions of equation (1)) that played a key role in the above mentioned Barouch-McCoy-Tracy-Wu theory, and, since then, it has appeared in many problems of statistical mechanics and quantum field theory. Let us now remind some of the basic known facts about the asymptotics of the solutions of equation (1). We refer the reader to monograph [4] for more details and for the history of the question.

Equation (1) possesses a two-parameter family of solutions characterized by the following behavior at $x=0$,

$$
u(x)=\alpha \ln x+\beta+O\left(x^{2-|\Im \alpha|}\right), \quad x \rightarrow 0,
$$

where the complex numbers $\alpha \in \mathbb{C},|\Im \alpha|<2$ and $\beta \in \mathbb{C}$ can be taken as parameters - the Cauchy data, of the solution $u(x) \equiv u(x \mid \alpha, \beta)$. The behavior of the solution $u(x \mid \alpha, \beta)$ as $x \rightarrow+\infty$, is known. For an open set in the space of parameters $\alpha, \beta$, which we will describe later, the large $x$ behavior of $u(x \mid \alpha, \beta)$ is oscillatory, and it is given by the formulae,

$$
\begin{aligned}
& u(x)=b_{+} e^{i x} x^{i \nu-1 / 2}\left(1+O\left(\frac{1}{x}\right)\right)+b_{-} e^{-i x} x^{-i \nu-1 / 2}\left(1+O\left(\frac{1}{x}\right)\right)+ \\
& +O\left(x^{3|\Im \nu|-3 / 2}\right)(\bmod 2 \pi), \quad x \rightarrow \infty,
\end{aligned}
$$

where

$$
\nu=-\frac{1}{4} b_{+} b_{-}, \quad|\Im \nu|<1 / 2 .
$$

The asymptotic parameters at infinity - the complex amplitudes $b_{ \pm}$, can be, in fact, expressed in terms of the Cauchy data $\alpha, \beta$, and the condition $|\Im \nu|<1 / 2$ imposes additional restriction on them. The corresponding connection formulae were obtained in 1985 by V. Yu. Novokshenov [18] (see also: [10] and [11]), and they are given by the equations,

$$
e^{\pi \nu}=\frac{\sin 2 \pi \eta}{\sin 2 \pi \sigma}, \quad b_{ \pm}=-e^{\frac{\pi \nu}{2} \mp \frac{i \pi}{4}} 2^{1 \pm 2 i \nu} \frac{1}{\sqrt{2 \pi}} \Gamma(1 \mp i \nu) \frac{\sin 2 \pi(\sigma \mp \eta)}{\sin 2 \pi \eta}
$$

where

$$
\sigma:=\frac{1}{4}+\frac{i}{8} \alpha, \quad \eta:=\frac{1}{4}+\frac{1}{4 \pi}(\beta+\alpha \ln 8)+\frac{i}{2 \pi} \ln \frac{\Gamma\left(\frac{1}{2}-\frac{i \alpha}{4}\right)}{\Gamma\left(\frac{1}{2}+\frac{i \alpha}{4}\right)},
$$


and $\Gamma(z)$ is Euler's Gamma-function. The open set in the space of the Cauchy data $\alpha, \beta$ where the both asymptotics, (4) and (5) are valid is described by the inequalities (see also Remark 2 below),

$$
0<\Re \sigma<\frac{1}{2} \Longleftrightarrow|\Im \alpha|<2, \quad \sin 2 \pi \eta \neq 0, \quad\left|\arg \frac{\sin 2 \pi \eta}{\sin 2 \pi \sigma}\right|<\frac{\pi}{2} \Longleftrightarrow|\Im \nu|<\frac{1}{2},
$$

where $\sigma$ and $\eta$ are understood as functions of $\alpha$ and $\beta$ defined in (8). We notice that this set contains all sufficiently small pairs $(\alpha, \beta)$, all real pairs $(\alpha, \beta)$ such that the corresponding $\eta$ satisfies the inequality $0<\eta<\frac{1}{2}(\bmod (1))$ and all pure imaginary pairs such that $|\alpha|<2$. In fact, it is convenient to take $\sigma$ and $\eta$ as the independent parameters and think about $\alpha$ and $\beta$ as their functions, i.e.,

$$
\alpha=i(2-8 \sigma), \quad \beta=-\pi+4 \pi \eta-i(2-8 \sigma) \ln 8-2 i \ln \frac{\Gamma(1-2 \sigma)}{\Gamma(2 \sigma)},
$$

where $\sigma, \eta$ are the complex numbers satisfying $(9)$. The expressions of the asymptotic parameters at $x=\infty$ in terms of $\sigma$ and $\eta$ have already been presented in (7).

The derivation of formulae (7) is based on the Isomonodromy-Riemann-Hilbert Method. We again refer the reader to monograph [4] for more details and for general references concerning the connection problem for Painlevé equations. In the framework of the Riemann-Hilbert method, the parameters $\sigma$ and $\eta$ have an independent important meaning as the monodromy data of the auxiliary linear system associated with the third Painlevé equation. This meaning of the parameters $\sigma$ and $\eta$ plays important role in the considerations of this paper, and it will be explained in detail in the next section.

Equations (4) and (5) in turn imply the following behavior at zero and at infinity of the corresponding tau-function (see also [8]),

$$
\tau(x)=C_{0} x^{-\frac{\alpha^{2}}{8}}(1+o(1)), \quad x \rightarrow 0,
$$

and

$$
\tau(x)=C_{\infty} x^{\nu^{2}} e^{\frac{x^{2}}{8}+2 \nu x}(1+o(1)), \quad x \rightarrow \infty .
$$

In fact, one can write a complete asymptotic series for the tau-function at both critical points whose coefficients are explicit functions of the Cauchy data $\alpha, \beta$ or, equivalently, of the monodromy data $\sigma, \eta$. The issue which we are concerned with is the evaluation of the ratio

$$
C_{\infty} / C_{0}
$$

in terms of the initial data $\alpha, \beta$. This can not be done just by using the asymptotic equations (4) - (5) and the connection formulae (7)-(8). Indeed, we are dealing here with the "constant of integration" problem. For the special one-parameter family of solutions of equation (1) related to the Ising model, this problem was solved by C. Tracy [20] in 1991. This special family is obtained by putting

$$
\eta=0 \quad \text { and } \quad \sigma \in \mathbb{R}, \quad 0<\sigma<\frac{1}{2}
$$


in (10). Zero value of $\eta$ is excluded from set (9) which means that the behavior of this special family at infinity is very different from the oscillatory one given in (5). In fact, all the solutions from this family exponentially approach $\pi(\bmod 2 \pi)$,

$$
u(x)-\pi \sim i \kappa \sqrt{\frac{2}{\pi}} x^{-1 / 2} e^{-x}, \quad x \rightarrow \infty, \quad \kappa=-2 \cos 2 \pi \sigma .
$$

In his calculations, Tracy made use of the existence of an additional Fredholm determinant representation of the tau-function evaluated on the family (14). We are interested in a generic, two-parameter case where there is no such representation. A conjectural answer to the problem has been produced in [12] with the help of the recently discovered by O. Gamayun, N. Iorgov, and O. Lisovyy connection of the Painlevé tau-functions with the Virasoro conformal blocks [5], [6]. In this paper we prove the conjecture of [12]. Our main result is the following theorem.

Theorem 1. Let $\sigma$ and $\eta$ be the "monodromy" parameters of the Painlevé III function $u(x)$ satisfying the inequalities (9). Then the ratio (13) is given by the formula,

$$
\begin{gathered}
\frac{C_{\infty}}{C_{0}}=\frac{2^{\frac{3}{2}} e^{-i \frac{\pi}{4}}}{\pi\left(G\left(\frac{1}{2}\right)\right)^{4}}(2 \pi)^{i \nu} 2^{2 \nu^{2}+\sigma^{2} 24-12 \sigma} e^{2 \pi i\left(\eta^{2}-2 \sigma \eta-\sigma^{2}+2 \eta-\sigma\right)} \\
\times \frac{\Gamma(1-2 \sigma)}{\Gamma(2 \sigma)}\left(\frac{G(1+i \nu) G(1+2 \sigma) G(1-2 \sigma) G\left(1+\sigma+\eta+\frac{1-i \nu}{2}\right) G\left(\frac{1-i \nu}{2}-\sigma-\eta\right)}{G\left(1+\sigma+\eta+\frac{1+i \nu}{2}\right) G\left(\frac{1+i \nu}{2}-\sigma-\eta\right)}\right)^{2},
\end{gathered}
$$

where $\nu$ is defined in (7) and $G(z)$ is the Barnes $G$ - function.

It should be noticed that in [12] a slightly different definition of the tau-function is used. The exact relation of the constant (15) and the one conjectured in [12] is discussed in the last section of the paper.

Our proof of Theorem 1 is not based on the conformal block connection. We use the Riemann-Hilbert representation of the third Painlevé transcendent and the Malgrange-Bertola extension of the Jimbo-Miwa-Ueno definition of the tau-function.

In the course of our proof, we also confirm one of the key observations of [12] that ratio (15) determines the generating function of the canonical transformation of the canonical variables determined by the initial data $(\alpha, \beta)$ to the canonical variables determined by the asymptotic data $\left(b_{+}, b_{-}\right)$(see the end of Section 5 for more detail). In fact, this Hamiltonian interpretation of the pre-factors in the asymptotics of the Painlevé tau-functions was first suggested in the work [7] of N. Iorgov, O. Lisovyy and Yu. Tykhyy.

The evaluation of ratio (13), which we have made rigorous in this paper, is only one of a series of highly nontrivial predictions and already established facts which came from the remarkable discovery of Gamayun, Iorgov, and Lisovyy. These other predictions and results, including the key ingredient of the approach of [5] and [6], which is the explicit conformal block series representations for the Painlevé tau-functions, do not yet have their understanding in the framework of the Riemann-Hilbert method.

We shall start the proof of Theorem 1 with the reminding of the Isomonodromy-RiemannHilbert formalism for the Painlevé equation (1) (for more detail see, e.g., [4]). 


\section{The Riemann-Hilbert Representation of the Solutions of the Sine-Gordon/Painlevé III Equation}

The Riemann-Hilbert problem associated with equation (1) is posed on the oriented contour $\Gamma$ depicted in Figure 1, and it consists in the finding of a $2 \times 2$ matrix-valued function $\Psi(\lambda)$ which satisfies the following properties.

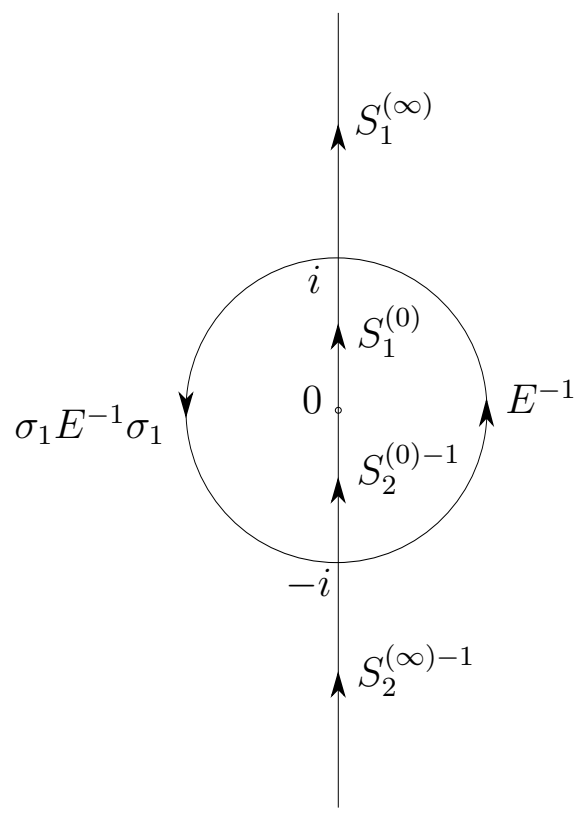

Figure 1: Contour $\Gamma$

- The function $\Psi(\lambda)$ is analytic on $\mathbb{C} \backslash\{\Gamma\}$, it has continuous \pm - limits on the contour $\Gamma$, and these limits satisfy jump condition $\Psi_{+}(\lambda)=\Psi_{-}(\lambda) S(\lambda)$. Here " + " denotes the boundary values from the left side of the contour and " - " denotes the boundary values from the right side of the contour. Jump matrix $S(\lambda)$ is piecewise constant, its different components are indicated in Figure 1, and they are given by the equations,

$$
\begin{aligned}
& S_{1}^{(\infty)}=S_{2}^{(0)}=\left(\begin{array}{cc}
1 & 0 \\
p+q & 1
\end{array}\right), \quad S_{2}^{(\infty)}=S_{1}^{(0)}=\left(\begin{array}{cc}
1 & p+q \\
0 & 1
\end{array}\right), \\
& E=\frac{1}{\sqrt{1+p q}}\left(\begin{array}{cc}
1 & p \\
-q & 1
\end{array}\right), \quad p, q \in \mathbb{C}, \quad 1+p q \neq 0, \quad \sigma_{1}=\left(\begin{array}{ll}
0 & 1 \\
1 & 0
\end{array}\right) .
\end{aligned}
$$

- The function $\Psi(\lambda)$ satisfies the following conditions at zero and infinity

$$
\begin{aligned}
& \Psi(\lambda)=P_{0}\left(I+M_{1}^{(0)} \lambda+O\left(\lambda^{2}\right)\right) e^{-\frac{i}{\lambda} \sigma_{3}}, \quad \lambda \rightarrow 0, \\
& \Psi(\lambda)=\left(I+\frac{M_{1}^{(\infty)}}{\lambda}+O\left(\frac{1}{\lambda^{2}}\right)\right) e^{-\frac{i x^{2} \lambda}{16} \sigma_{3}}, \quad \lambda \rightarrow \infty,
\end{aligned}
$$


where $P_{0}, M_{1}^{(0)}, M_{1}^{(\infty)}$ here are some constant in $\lambda$ matrices and

$$
\sigma_{3}=\left(\begin{array}{cc}
1 & 0 \\
0 & -1
\end{array}\right)
$$

The Riemann-Hilbert problem is uniquely and meromorphically in $x$ solvable for all $p, q \in \mathbb{C}, \quad 1+p q \neq 0$ [17] and the corresponding solution $u(x) \equiv u(x ; p, q)$ of the third Painlevé equation (1) is given by the formula,

$$
u(x)=2 \arccos \left(P_{0}\right)_{11} .
$$

In fact, the following equation takes place,

$$
P_{0}=\left(\begin{array}{cc}
\cos \left(\frac{u}{2}\right) & -i \sin \left(\frac{u}{2}\right) \\
-i \sin \left(\frac{u}{2}\right) & \cos \left(\frac{u}{2}\right)
\end{array}\right) \equiv e^{-\frac{i u \sigma_{1}}{2}} .
$$

Remark 1. The above Riemann-Hilbert setting corresponds to the generic solutions of (1). There is one parameter family of a separatrix solution which is characterized by the following Riemann-Hilbert data

$$
S_{1}^{(\infty)}=S_{2}^{(0)}=\left(\begin{array}{ll}
1 & 0 \\
\kappa & 1
\end{array}\right), \quad S_{2}^{(\infty)}=S_{1}^{(0)}=\left(\begin{array}{ll}
1 & \kappa \\
0 & 1
\end{array}\right), \quad E= \pm i\left(\begin{array}{ll}
0 & 1 \\
1 & 0
\end{array}\right), \quad \kappa \in \mathbb{C} .
$$

This is the family which includes the McCoy-Tracy-Wu solution (14) and which is not considered in this paper. As it has already been mentioned, the constant problem for this family was solved in [20].

The parameters $p, q \in \mathbb{C}$ in $(16),(17)$ are connected with the parameters of asymptotic of $u(x)$ via

$$
p:=-i \frac{\sin 2 \pi(\sigma+\eta)}{\sin 2 \pi \eta}, \quad q:=i \frac{\sin 2 \pi(\sigma-\eta)}{\sin 2 \pi \eta} .
$$

Conditions $|\Im \alpha|<2$ and $|\Im \nu|<1 / 2$ can be rewritten in terms of $p$ and $q$ as

$$
p+q \notin(-i \infty,-2 i] \cup[2 i,+i \infty), \quad \text { and } \quad p q \notin(-\infty,-1],
$$

respectively.

Remark 2. Conditions (20) are the conditions which appear during the asymptotic analysis of the Riemann-Hilbert problem. The asymptotic parameter $\nu$ is related to $p, q$ according to the equation,

$$
\nu=-\frac{1}{2 \pi} \ln (1+p q)
$$

Also,

$$
1+p q=\frac{\sin ^{2} 2 \pi \sigma}{\sin ^{2} 2 \pi \eta}
$$

We restrict ourselves in (9) to the inequality $\left|\arg \frac{\sin 2 \pi \eta}{\sin 2 \pi \sigma}\right|<\pi / 2$, instead of the inequality $\left|\arg \left(\frac{\sin 2 \pi \eta}{\sin 2 \pi \sigma}\right)^{2}\right|<\pi$ by a technical reason. This means that we actually analyze one of the 
components of the full set of the Cauchy data corresponding to the generic asymptotic behavior (4) and (5). The another component is defined by the condition,

$$
\left|\arg \frac{\sin 2 \pi \eta}{\sin 2 \pi \sigma}-\pi\right|<\pi / 2
$$

which in turn implies the following change in formulae (7),

$$
e^{\pi \nu}=-\frac{\sin 2 \pi \eta}{\sin 2 \pi \sigma}
$$

The analysis presented in this paper can be easily extended on this component of initial data as well. Actually, the $\tau$-function does not change if we add $2 \pi i$ to the function $u(x)$. But $\eta$ is shifted by $\frac{1}{2}$. So such change of variable allows us to go from one component to another.

Function $\Psi(\lambda)$ satisfies system of linear ordinary differential equations

$$
\begin{aligned}
& \frac{d \Psi}{d \lambda}=A(\lambda) \Psi(\lambda) \\
& \frac{d \Psi}{d x}=U(\lambda) \Psi(\lambda) \\
& A(\lambda)=-\frac{i x^{2} \sigma_{3}}{16}-\frac{i x u_{x} \sigma_{1}}{4 \lambda}+\frac{P_{0}\left(i \sigma_{3}\right) P_{0}^{-1}}{\lambda^{2}}, \\
& U(\lambda)=-\frac{i \lambda x \sigma_{3}}{8}-\frac{i u_{x} \sigma_{1}}{2} .
\end{aligned}
$$

Equation (1) is the compatibility condition for this system and it describes isomonodromic deformations of the system (21). From this point of view $\sigma$ and $\eta$ play role of the monodromy data.

We complete this overview of the Riemann-Hilbert formalism for equation (1) by presenting the general alternative definition of the Jimbo-Miwa-Ueno tau-function in terms of the solution $\Psi(\lambda)$ of the Riemann-Hilbert problem.

Define

$$
\hat{\Psi}^{(\infty)}(\lambda):=\Psi(\lambda) e^{\frac{i x^{2} \lambda}{16} \sigma_{3}}, \quad|\lambda|>R .
$$

Then, according to [9] the equation,

$$
\omega_{J M U}=-\underset{\lambda=\infty}{\operatorname{res}} \operatorname{Tr}\left(\left(\hat{\Psi}^{(\infty)}(\lambda)\right)^{-1}\left(\hat{\Psi}^{(\infty)}(\lambda)\right)^{\prime}\left(-\frac{i \lambda x}{8} \sigma_{3}\right)\right) d x,
$$

defines the differential form whose antiderivative is the logarithm of tau-function. Actually, from (18) we have that

$$
\hat{\Psi}^{(\infty)}(\lambda)=I+\frac{M_{1}^{(\infty)}}{\lambda}+O\left(\frac{1}{\lambda^{2}}\right) .
$$

Substituting (18) to the equation (21), one can express $M_{1}^{(\infty)}$ in terms of $u(x)$ (see [4],[17]).

$$
M_{1}^{(\infty)}=-\frac{2 i x u_{x}}{x^{2}} \sigma_{2}-i\left(\cos u-\frac{u_{x}^{2}}{2}\right) \sigma_{3}, \quad \sigma_{2}=\left(\begin{array}{cc}
0 & -i \\
i & 0
\end{array}\right)
$$


Substituting, in turn, (24)-(25) into (23) yields the relation, $\omega_{J M U}=d \ln \tau(x)$, where $d \ln \tau$ is defined in (3). In other words, the tau-function can be alternatively defined as

$$
\tau \equiv \tau_{J M U}(x, p, q)=e^{\int \omega_{J M U}} .
$$

So defined the tau-function is unique up to multiplication by a constant depending on $p$ and $q$. The key fact for us is that, following [2], it is possible to extend Jimbo-Miwa-Ueno differential form (23) on vector fields in $p$ and $q$ in such a way, that it will remains a closed form. Such extension will allow us to define tau-function already up to a constant, which does not depend on $p$ and $q$.

\section{Malgrange-Bertola Differential Form}

In this section we basically repeat the calculations and the results of Section 5.1 of paper [2] adjusting them to our special case.

Put $Y(\lambda)=\Psi(\lambda) e^{\left(\frac{i x^{2} \lambda}{16}+\frac{i}{\lambda}\right) \sigma_{3}}$. Denote $G(\lambda)$ the jump matrix for $Y(\lambda)$. Following [2], [14], we define the Malgrange-Bertola differential form by the equation

$$
\omega_{M B}[\partial]=\int_{\Gamma} \operatorname{Tr}\left(Y_{-}^{-1} Y_{-}^{\prime}(\partial G) G^{-1} \frac{d \lambda}{2 \pi i} .\right.
$$

Here $\partial$ denotes the vector field in the space of parameters $x, p, q$, and the prime denotes derivative with respect to $\lambda$. This differential form was introduced originally by B. Malgrange in [14] for the case when the contour $\Gamma$ is a circle. M. Bertola in [2] has extended the Malgrange's definition to an arbitrary Riemann-Hilbert setting.

Let us establish the connection of this form with Jimbo-Miwa-Ueno form. For the case of general Riemann-Hilbert problem, the analog of this Lemma was proven in [2].

Lemma 1. The Malgrange-Bertola differential form, evaluated on the vector fields in parameter $x$, is equal to the Jimbo-Miwa-Ueno form up to a term, depending only on $G(\lambda)$.

$$
\omega_{M B}\left[\partial_{x}\right]=\omega_{J M U}\left[\partial_{x}\right]-\left[\int_{\Gamma} \operatorname{Tr}\left(G^{-1} G^{\prime} \frac{i x \lambda}{8} \sigma_{3}\right) \frac{d \lambda}{2 \pi i}\right]-\frac{x}{4} .
$$

Proof. First, we have

$$
G(\lambda)=e^{-\left(\frac{i x^{2} \lambda}{16}+\frac{i}{\lambda}\right) \sigma_{3}} S(\lambda) e^{\left(\frac{i x^{2} \lambda}{16}+\frac{i}{\lambda}\right) \sigma_{3}},
$$

where $S(\lambda)$ is the jump matrix for $\Psi(\lambda)$. Hence,

$$
\partial_{x} G(\lambda) G^{-1}(\lambda)=-\frac{i x \lambda}{8} \sigma_{3}+\frac{i x \lambda}{8} G(\lambda) \sigma_{3} G^{-1}(\lambda) .
$$

Also, we have that

$$
Y_{+}(\lambda)=Y_{-}(\lambda) G(\lambda),
$$

and, by $\lambda$ - differentiation,

$$
Y_{+}^{\prime}(\lambda)=Y_{-}^{\prime}(\lambda) G(\lambda)+Y_{-}(\lambda) G^{\prime}(\lambda) .
$$


Substituting (28), 29), 30) in (26) we get

$$
\omega_{M B}\left[\partial_{x}\right]=\left[\int_{\Gamma} \operatorname{Tr}\left(\left(Y_{+}^{-1} Y_{+}^{\prime}-Y_{-}^{-1} Y_{-}^{\prime}\right) \frac{i x \lambda}{8} \sigma_{3}-G^{-1} G^{\prime} \frac{i x \lambda}{8} \sigma_{3}\right) \frac{d \lambda}{2 \pi i}\right] .
$$

Let us introduce the notation for the parts of the contour $\Gamma$ as it is indicated in Figure 2 ,

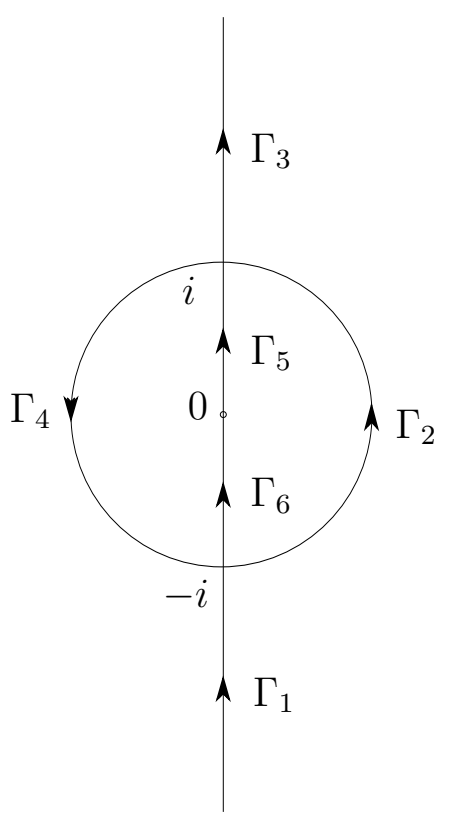

Figure 2: Parts of the contour $\Gamma$ 
We have

$$
\begin{gathered}
\int_{\Gamma_{5} \cup \Gamma_{6} \cup \Gamma_{4}} \operatorname{Tr}\left(Y_{+}^{-1} Y_{+}^{\prime} \frac{i x \lambda}{8} \sigma_{3}\right) \frac{d \lambda}{2 \pi i}=0 \\
\int_{\Gamma_{2}} \operatorname{Tr}\left(Y_{+}^{-1} Y_{+}^{\prime} \frac{i x \lambda}{8} \sigma_{3}\right) \frac{d \lambda}{2 \pi i}-\int_{\Gamma_{5} \cup \Gamma_{6}} \operatorname{Tr}\left(Y_{-}^{-1} Y_{-}^{\prime} \frac{i x \lambda}{8} \sigma_{3}\right) \frac{d \lambda}{2 \pi i}=0 .
\end{gathered}
$$

We also have

$$
Y(\lambda)=\hat{\Psi}^{(\infty)}(\lambda) e^{\frac{i}{\lambda} \sigma_{3}}, \quad|\lambda|>R .
$$

Hence,

$$
\begin{aligned}
& \int_{\Gamma_{1} \cup \Gamma_{3}} \operatorname{Tr}\left(Y_{+}^{-1} Y_{+}^{\prime} \frac{i x \lambda}{8} \sigma_{3}\right) \frac{d \lambda}{2 \pi i}-\int_{\Gamma_{1} \cup \Gamma_{2} \cup \Gamma_{3} \cup \Gamma_{4}} \operatorname{Tr}\left(Y_{-}^{-1} Y_{-}^{\prime} \frac{i x \lambda}{8} \sigma_{3}\right) \frac{d \lambda}{2 \pi i} \\
& =\underset{\lambda=\infty}{\operatorname{res}} \operatorname{Tr}\left(e^{-\frac{i}{\lambda} \sigma_{3}}\left(\hat{\Psi}^{(\infty)}(\lambda)\right)^{-1}\left(\hat{\Psi}^{(\infty)}(\lambda)\right)^{\prime} e^{\frac{i}{\lambda} \sigma_{3}} \frac{i x \lambda}{8} \sigma_{3}-e^{-\frac{i}{\lambda} \sigma_{3}}\left(\hat{\Psi}^{(\infty)}(\lambda)\right)^{-1}\left(\hat{\Psi}^{(\infty)}(\lambda)\right) \frac{i}{\lambda^{2}} \sigma_{3} e^{\frac{i}{\lambda} \sigma_{3}} \frac{i x \lambda}{8} \sigma_{3}\right) \\
& =\underset{\lambda=\infty}{\operatorname{res}} \operatorname{Tr}\left(\left(\hat{\Psi}^{(\infty)}(\lambda)\right)^{-1}\left(\hat{\Psi}^{(\infty)}(\lambda)\right)^{\prime} \frac{i x \lambda}{8} \sigma_{3}\right)-\frac{x}{4},
\end{aligned}
$$

and (27) follows.

This lemma means, that Malgrange-Bertola form is indeed a good candidate for extension of Jimbo-Miwa-Ueno form. However, there is an additional term, depending only on $G(\lambda)$. One can, following again [2], cancel it considering the modified Malgrange-Bertola form $\omega=\omega_{M B}+\theta$, where

$$
\theta[\partial]=\frac{1}{2} \int_{\hat{\Gamma}} \operatorname{Tr}\left(G^{\prime} G^{-1}(\partial G) G^{-1}\right) \frac{d \lambda}{2 \pi i} .
$$

In the notations of [2] $\omega$ is the form $\Omega$ from Definition 2.2 of [2].

We have $\omega\left[\partial_{x}\right]=\omega_{J M U}\left[\partial_{x}\right]-\frac{x}{4}$. Indeed,

$$
\begin{gathered}
G^{-1} G^{\prime} \frac{i x \lambda}{8} \sigma_{3}=\frac{i x \lambda}{8}\left(\frac{i x^{2}}{16}-\frac{i}{\lambda^{2}}\right)\left(I-G^{-1} \sigma_{3} G \sigma_{3}\right), \\
\operatorname{Tr}\left(G^{\prime} G^{-1}\left(\partial_{x} G\right) G^{-1}\right)=\frac{i x \lambda}{8}\left(\frac{i x^{2}}{16}-\frac{i}{\lambda^{2}}\right) 2 \operatorname{Tr}\left(I-G^{-1} \sigma_{3} G \sigma_{3}\right),
\end{gathered}
$$

and the additional term depending only on $G(\lambda)$ cancels.

In the next section we will express the form $\omega$ in terms of the coefficients of the asymptotic expansions of $Y(\lambda)$ at $\lambda=0$ and at $\lambda=\infty$. We call this expression a "localization" of the original integral formula (26) for the Malgrange-Bertola form. This localized version will simplify dramatically the further analysis of the form $\omega$.

\section{Localization}

Let us introduce the function

$$
\Theta(\lambda)=\partial Y(\lambda) Y(\lambda)^{-1}
$$


where $\partial$ means the differentiation with respect to one of the three parameters, $x, p$, or $q$. The $\partial$-version of equation 30 reads,

$$
\partial Y_{+}(\lambda)=\partial Y_{-}(\lambda) G(\lambda)+Y_{-}(\lambda) \partial G(\lambda) .
$$

Expressing $G(\lambda)$ from 29$)$ as $G(\lambda)=Y_{-}^{-1}(\lambda) Y_{+}(\lambda)$ we rewrite the last equations as

$$
\partial Y_{+}(\lambda) Y_{+}^{-1}(\lambda)=\partial Y_{-}(\lambda) Y_{-}^{-1}(\lambda)+Y_{-}(\lambda) \partial G(\lambda) Y_{+}^{-1}(\lambda)
$$

or as

$$
\partial Y_{+}(\lambda) Y_{+}^{-1}(\lambda)-\partial Y_{-}(\lambda) Y_{-}^{-1}(\lambda)=Y_{-}(\lambda) \partial G(\lambda) Y_{+}^{-1}(\lambda) .
$$

The Sokhotski-Plemelj formula would then imply (cf. Lemma 2.1 of [2]) that

$$
\Theta(\lambda)=\int_{\Gamma} \frac{Y_{-}(y) \partial G(y) Y_{+}^{-1}(y)}{y-\lambda} \frac{d y}{2 \pi i} .
$$

Substituting $Y(\lambda)=\Psi(\lambda) e^{\left(\frac{i x^{2} \lambda}{16}+\frac{i}{\lambda}\right) \sigma_{3}}$ in $(21)$, we have

$$
Y^{\prime}(\lambda)=A(\lambda) Y(\lambda)+\left(\frac{i x^{2}}{16}-\frac{i}{\lambda^{2}}\right) Y(\lambda) \sigma_{3},
$$

and

$$
\begin{aligned}
& \omega_{M B}[\partial]=\int_{\Gamma} \operatorname{Tr}\left(Y_{-}^{-1} Y_{-}^{\prime}(\partial G) G^{-1}\right) \frac{d \lambda}{2 \pi i}=\int_{\Gamma}\left(\frac{i x^{2}}{16}-\frac{i}{\lambda^{2}}\right) \operatorname{Tr}\left(\sigma_{3}(\partial G) G^{-1}\right) \frac{d \lambda}{2 \pi i} \\
& +\int_{\Gamma} \operatorname{Tr}\left(A Y_{-}(\partial G) Y_{+}^{-1}\right) \frac{d \lambda}{2 \pi i} .
\end{aligned}
$$

We introduce notation

$$
\begin{aligned}
& Y(\lambda)=P_{0}\left(I+\stackrel{\circ}{m}_{1} \lambda+O\left(\lambda^{2}\right)\right), \quad \lambda \rightarrow 0, \\
& Y(\lambda)=\left(I+\frac{m_{1}^{(\infty)}}{\lambda}+O\left(\frac{1}{\lambda^{2}}\right)\right), \quad \lambda \rightarrow \infty .
\end{aligned}
$$

Substituting these expressions for $Y(\lambda)$ to the definition of $\Theta(\lambda)$ we get

$$
\begin{gathered}
\Theta(\lambda)=\left(\partial P_{0}\right) P_{0}^{-1}+P_{0}\left(\partial \stackrel{\circ}{m}_{1}\right) P_{0}^{-1} \lambda+O\left(\lambda^{2}\right), \quad \lambda \rightarrow 0, \\
\Theta(\lambda)=\frac{\partial m_{1}^{(\infty)}}{\lambda}+O\left(\frac{1}{\lambda^{2}}\right), \quad \lambda \rightarrow \infty .
\end{gathered}
$$

Comparing these formulae with (32) we arrive at the relations

$$
\int_{\Gamma} Y_{-}(y) \partial G(y) Y_{+}^{-1}(y) \frac{d y}{2 \pi i}=-\partial m_{1}^{(\infty)},
$$




$$
\begin{aligned}
& \int_{\Gamma} \frac{Y_{-}(y) \partial G(y) Y_{+}^{-1}(y)}{y} \frac{d y}{2 \pi i}=\left(\partial P_{0}\right) P_{0}^{-1}, \\
& \int_{\Gamma} \frac{Y_{-}(y) \partial G(y) Y_{+}^{-1}(y)}{y^{2}} \frac{d y}{2 \pi i}=P_{0}\left(\partial \stackrel{\circ}{m}_{1}\right) P_{0}^{-1} .
\end{aligned}
$$

Let us look now at the last integral in equation (34). Putting in it formula 22 for $A(\lambda)$, we will see that this integral can be re-written as

$$
\begin{gathered}
\int_{\Gamma} \operatorname{Tr}\left(A Y_{-}(\partial G) Y_{+}^{-1}\right) \frac{d \lambda}{2 \pi i}=-\frac{i x^{2}}{16} \int_{\Gamma} \operatorname{Tr}\left(\sigma_{3} Y_{-}(\partial G) Y_{+}^{-1}\right) \frac{d \lambda}{2 \pi i} \\
-\frac{i x u_{x}}{4} \int_{\Gamma} \operatorname{Tr}\left(\sigma_{1} \frac{Y_{-}(\partial G) Y_{+}^{-1}}{\lambda}\right) \frac{d \lambda}{2 \pi i}+\int_{\Gamma} \operatorname{Tr}\left(P_{0}\left(i \sigma_{3}\right) P_{0}^{-1} \frac{Y_{-}(\partial G) Y_{+}^{-1}}{\lambda^{2}}\right) \frac{d \lambda}{2 \pi i} \\
=-\frac{i x^{2}}{16} \operatorname{Tr}\left(\sigma_{3} \int_{\Gamma} Y_{-}(\partial G) Y_{+}^{-1} \frac{d \lambda}{2 \pi i}\right) \\
-\frac{i x u_{x}}{4} \operatorname{Tr}\left(\sigma_{1} \int_{\Gamma} \frac{Y_{-}(\partial G) Y_{+}^{-1}}{\lambda} \frac{d \lambda}{2 \pi i}\right)+\operatorname{Tr}\left(P_{0}\left(i \sigma_{3}\right) P_{0}^{-1} \int_{\Gamma} \frac{Y_{-}(\partial G) Y_{+}^{-1}}{\lambda^{2}} \frac{d \lambda}{2 \pi i}\right) .
\end{gathered}
$$

The last equation, with the help of (37) - (39), is transformed into the localized formula,

$$
\int_{\Gamma} \operatorname{Tr}\left(A Y_{-}(\partial G) Y_{+}^{-1}\right) \frac{d \lambda}{2 \pi i}=\frac{i x^{2}}{16} \operatorname{Tr}\left(\sigma_{3} \partial m_{1}^{(\infty)}\right)-\frac{i x u_{x}}{4} \operatorname{Tr}\left(\sigma_{1}\left(\partial P_{0}\right) P_{0}^{-1}\right)+i \operatorname{Tr}\left(\sigma_{3} \partial \dot{\circ}_{1}\right) .
$$

Substituting the derivative of $G$ with respect to $\lambda$ in the formula for $\theta$, we have that

$$
\theta[\partial]=\frac{1}{2} \int_{\Gamma}\left(\frac{i x^{2}}{16}-\frac{i}{\lambda^{2}}\right) \operatorname{Tr}\left(\sigma_{3}\left(G^{-1} \partial G-(\partial G) G^{-1}\right)\right) \frac{d \lambda}{2 \pi i} .
$$

Together with 40 this gives us the following formula for $\omega$

$$
\begin{gathered}
\omega[\partial]=\frac{1}{2} \int_{\Gamma}\left(\frac{i x^{2}}{16}-\frac{i}{\lambda^{2}}\right) \operatorname{Tr}\left(\sigma_{3}\left((\partial G) G^{-1}+G^{-1} \partial G\right)\right) \frac{d \lambda}{2 \pi i} \\
+\frac{i x^{2}}{16} \operatorname{Tr}\left(\sigma_{3} \partial m_{1}^{(\infty)}\right)-\frac{i x u_{x}}{4} \operatorname{Tr}\left(\sigma_{1}\left(\partial P_{0}\right) P_{0}^{-1}\right)+i \operatorname{Tr}\left(\sigma_{3} \partial \stackrel{\circ}{m_{1}}\right) .
\end{gathered}
$$

One can check directly that

$$
\operatorname{Tr}\left(\sigma_{3}\left((\partial G) G^{-1}+G^{-1}(\partial G)\right)\right) \equiv 0 .
$$


Therefore, we finally have that

$$
\omega[\partial]=\frac{i x^{2}}{16} \operatorname{Tr}\left(\sigma_{3} \partial m_{1}^{(\infty)}\right)-\frac{i x u_{x}}{4} \operatorname{Tr}\left(\sigma_{1}\left(\partial P_{0}\right) P_{0}^{-1}\right)+i \operatorname{Tr}\left(\sigma_{3} \partial \stackrel{\circ}{m_{1}}\right) .
$$

Substituting (35), (36) to the equation (33), one can express the coefficients of asymptotics of $Y(\lambda)$ at $\lambda=0$ and at $\lambda=\infty$ in terms of $u$. In particular, one gets (cf. [17]),

$$
\begin{aligned}
& m_{1}^{(\infty)}=-\frac{2 i x u_{x}}{x^{2}} \sigma_{2}-i\left(\cos u-1-\frac{u_{x}^{2}}{2}\right) \sigma_{3}, \\
& \stackrel{\circ}{m}_{1}=\frac{i x u_{x}}{8} \sigma_{2}-i\left(\frac{x^{2}}{16}(\cos u-1)-\frac{x^{2} u_{x}^{2}}{32}\right) \sigma_{3} .
\end{aligned}
$$

Inserting these equations together with formula $(19)$ in (41) and using also the fact, that $u(x)$ satisfies (1) we transform equation (41) into the final expression for the form $\omega$ in terms of $u$ and its derivatives with respect to $x, p$ and $q$.

Proposition 1. The modified Malgrange-Bertola differential form $\omega$ admits the following representation

$$
\begin{aligned}
& \omega=\left(-\frac{x u_{x}^{2}}{8}+\frac{x}{4}(\cos u-1)\right) d x-\left(\frac{x^{2}}{4} u_{p} \sin u+\frac{x^{2}}{4} u_{x} u_{p x}+\frac{x u_{x} u_{p}}{4}\right) d p \\
& -\left(\frac{x^{2}}{4} u_{q} \sin u+\frac{x^{2}}{4} u_{x} u_{q x}+\frac{x u_{x} u_{q}}{4}\right) d q .
\end{aligned}
$$

We notice that from (42) we have again the statement of Lemma 1, that is that $\omega\left[\partial_{x}\right]=$ $\partial_{x} \ln \tau-\frac{x}{4}$. We want to mention again that this part of the localization formulae has already been obtained in [2]. We also want to emphasize the important role which is played by the $\lambda$ - equation (21) in the derivation of the $p, q$ - part of equation (42). It is the use of this equation that allowed us to present the original Malgrange-Bertola integral (26), first in the form (34), and then in the localized form (40). In fact, similar technique has already been used in the study of Toeplitz determinants with the Fisher-Hartwig singularities in paper [3] - see Appendix 6 and Lemma 6.2 of that paper.

Remark 3. As it was pointed out to the authors by M. Bertola, equation (31) can be used in the derivations of this section one more time and help to make a significant short cut from equation (34) to the localized form (40). Indeed, Bertola's suggestion is to use relation (31) for the product $Y_{-}(\lambda) G(\lambda) Y_{+}^{-1}(\lambda)$ in the last integral of (34) directly and rewrite this integral as

$$
\begin{aligned}
& \int_{\Gamma} \operatorname{Tr}\left(A(\lambda) Y_{-}(\lambda) \partial G(\lambda) Y_{+}^{-1}(\lambda)\right) \frac{d \lambda}{2 \pi i}=\int_{\Gamma} \operatorname{Tr}\left(A(\lambda)\left(\partial Y_{+}(\lambda) Y_{+}^{-1}(\lambda)-\partial Y_{-}(\lambda) Y_{-}^{-1}(\lambda)\right)\right) \frac{d \lambda}{2 \pi i} \\
& \quad=\sum_{\text {poles of } A(\lambda) d \lambda} \text { res } \operatorname{Tr}\left(A(\lambda) \partial Y(\lambda) Y^{-1}(\lambda)\right) .
\end{aligned}
$$

This is a quite general construction which allows one to localize the Malgrange-Bertola form for an arbitrary isomonodromic Riemann-Hilbert problem. In our case, one has to evaluate the, properly understood, residues at the points $\lambda=0, \infty$. The result will be equation (40). 


\section{Proof of Theorem 1}

Let us compute $d \omega$. First we have,

$d\left[\left(\frac{x u_{x}^{2}}{8}-\frac{x}{4}(\cos u-1)\right) d x\right]=\left(\frac{x u_{x} u_{p x}}{4}+\frac{x u_{p} \sin u}{4}\right) d p \wedge d x+\left(\frac{x u_{x} u_{q x}}{4}+\frac{x u_{q} \sin u}{4}\right) d q \wedge d x$.

Then, using the fact that $u(x)$ satisfies equation (1), we get that

$$
\begin{gathered}
d\left[\left(\frac{x^{2}}{4} u_{p} \sin u+\frac{x^{2}}{4} u_{x} u_{p x}+\frac{x u_{x} u_{p}}{4}\right) d p\right]=\left(\frac{x u_{x} u_{p x}}{4}+\frac{x u_{p} \sin u}{4}\right) d x \wedge d p \\
+\left(\frac{x^{2}}{4} u_{p q} \sin u+\frac{x^{2}}{4} u_{p} u_{q} \cos u+\frac{x^{2}}{4} u_{p x} u_{q x}+\frac{x^{2}}{4} u_{x} u_{x p q}+\frac{x}{4} u_{x} u_{p q}+\frac{x}{4} u_{p} u_{q x}\right) d q \wedge d p .
\end{gathered}
$$

and

$$
\begin{gathered}
d\left[\left(\frac{x^{2}}{4} u_{q} \sin u+\frac{x^{2}}{4} u_{x} u_{q x}+\frac{x u_{x} u_{q}}{4}\right) d q\right]=\left(\frac{x u_{x} u_{q x}}{4}+\frac{x u_{q} \sin u}{4}\right) d x \wedge d q \\
+\left(\frac{x^{2}}{4} u_{p q} \sin u+\frac{x^{2}}{4} u_{p} u_{q} \cos u+\frac{x^{2}}{4} u_{p x} u_{q x}+\frac{x^{2}}{4} u_{x} u_{x p q}+\frac{x}{4} u_{x} u_{p q}+\frac{x}{4} u_{q} u_{p x}\right) d p \wedge d q .
\end{gathered}
$$

Adding up the last three equations we obtain that

$$
d \omega=\frac{v_{p} u_{q}-v_{q} u_{p}}{4} d q \wedge d p,
$$

where $v=x u_{x}$. From equation (1) it follows that

$$
\frac{d}{d x}\left(v_{p} u_{q}-v_{q} u_{p}\right)=0
$$

and hence we can observe that

$$
d \omega=\lim _{x \rightarrow 0} d \omega=\frac{\alpha_{p} \beta_{q}-\alpha_{q} \beta_{p}}{4} d q \wedge d p=\frac{d \beta \wedge d \alpha}{4} .
$$

Therefore, if we define

$$
w=\omega+\frac{x}{4} d x+\frac{\alpha d \beta}{4}
$$

then the form $w$ will be a closed form on the full set of parameters, $(x, p, q)$ and such that $w\left[\partial_{x}\right]=w_{J M U}\left[\partial_{x}\right]$. This means we can put,

$$
\tau=e^{\int w}
$$

and this equation would define the tau-function up to a constant, which does not depend on $p$ and $q$.

Remark 4. It is worth noticing, that from our analysis it follows that, in the case of the Painlevé III equation (1), the external differential of the (modified) Malgrange-Bertola form $\omega$ is proportional to the canonical symplectic form (2) for the Hamiltonian dynamics of the Painlevé equation; indeed, we have that i.e.,

$$
d \omega=-\frac{1}{4} \Omega
$$


The next step is to evaluate the small and the large $x$ asymptotics of the form $w$. To this end we shall use asymptotics (4), (5) for $u(x)$ and make the following temporary technical assumptions,

$$
|\Im \nu|<\frac{1}{6}, \quad|\Im \alpha|<1 .
$$

In our calculations we will need more terms of the large $x$ asymptotics at infinity which are given in [12],

$$
\begin{gathered}
u(x)=b_{+} e^{i x} x^{i \nu-\frac{1}{2}}+b_{-} e^{-i x} x^{-i \nu-\frac{1}{2}} \\
+\frac{i b_{+}}{8}\left(6 \nu^{2}+4 i \nu-1\right) e^{i x} x^{i \nu-\frac{3}{2}}-\frac{i b_{-}}{8}\left(6 \nu^{2}-4 i \nu-1\right) e^{-i x} x^{-i \nu-\frac{3}{2}} \\
-\frac{1}{48} b_{+}^{3} e^{3 i x} x^{3 i \nu-\frac{3}{2}}-\frac{1}{48} b_{-}^{3} e^{-3 i x} x^{-3 i \nu-\frac{3}{2}}+O\left(x^{-\frac{5}{2}+5|\Im \nu|}\right) .
\end{gathered}
$$

Substituting this asymptotics at the right hand side of equation (42), we shall arrive, after rather tedious though straightforward calculations, at the following asymptotic representation of the form $\omega$ as $x \rightarrow \infty$,

$$
\begin{aligned}
& \omega=d\left(2 \nu x+\nu^{2} \ln x+\nu^{2}\right)-\frac{i}{4}\left(b_{+} d b_{-}-b_{-} d b_{+}\right)+\left(\frac{i b_{+}^{2}}{8} e^{2 i x} x^{2 i \nu-1}-\frac{i b_{-}^{2}}{8} e^{-2 i x} x^{-2 i \nu-1}\right) d x \\
& \left.\left.\quad+O\left(x^{-2+6|\Im \nu|}\right) d x+O\left(x^{-1+6|\Im \nu|}\right)\right) d p+O\left(x^{-1+6|\Im \nu|}\right)\right) d q, \quad x \rightarrow \infty
\end{aligned}
$$

The derivation of the small $x$ asymptotics of the form $\omega$ is based just on the estimate (4), i.e., no need for its extension, and it is much easy to obtain,

$$
\begin{array}{r}
\left.\omega=d\left(-\frac{\alpha^{2}}{8} \ln x-\frac{\alpha^{2}}{8}\right)-\frac{\alpha d \beta}{4}+O\left(x^{1-|\Im(\alpha)|}\right)\right) d x \\
\left.\left.+O\left(x^{2-|\Im(\alpha)|} \ln x\right)\right) d p+O\left(x^{2-|\Im(\alpha)|} \ln x\right)\right) d q, \quad x \rightarrow 0 .
\end{array}
$$

As it has already been indicated, the derivations of formulae (46) and (47) are straightforward. However, because of the importance of these formulae for our further analysis, we present the details of their derivations in the Appendix.

In view of the assumptions (44), estimates (46) and (47) yield the following asymptotic representation for the form $w$,

$$
w=-d\left(\frac{\alpha^{2}}{8} \ln x+\frac{\alpha^{2}}{8}\right)+o(1), \quad x \rightarrow 0,
$$

and

$$
w=d\left(2 \nu x+\nu^{2} \ln x+\nu^{2}\right)-\frac{i}{4}\left(b_{+} d b_{-}-b_{-} d b_{+}\right)+\frac{x}{4} d x+\frac{\alpha d \beta}{4}+o(1), \quad x \rightarrow \infty .
$$


On the other hand, from (11) and 12 we have that

$$
w=-d\left(\frac{\alpha^{2}}{8} \ln x\right)+d \ln C_{0}+o(1), \quad x \rightarrow 0,
$$

and

$$
w=d\left(2 \nu x+\nu^{2} \ln x+\frac{x^{2}}{8}\right)+d \ln C_{\infty}+o(1), \quad x \rightarrow \infty .
$$

The comparison of 48$)-(49)$ and $(50)$ - (51) implies that

$$
d \ln C_{0}=-d\left(\frac{\alpha^{2}}{8}\right)
$$

and

$$
d \ln C_{\infty}=d \nu^{2}-\frac{i}{4}\left(b_{+} d b_{-}-b_{-} d b_{+}\right)+\frac{\alpha d \beta}{4} .
$$

The last two equations mean that

$$
d \ln \frac{C_{\infty}}{C_{0}}=d\left(\nu^{2}+\frac{\alpha^{2}}{8}-i \nu\right)+\frac{\alpha d \beta}{4}-\frac{i}{2} b_{+} d b_{-}
$$

(where we have also taken into account (6)), or that

$$
\ln \frac{C_{\infty}}{C_{0}}=\nu^{2}+\frac{\alpha^{2}}{8}-i \nu+\frac{1}{4} \int\left(\alpha d \beta-2 i b_{+} d b_{-}\right)+c,
$$

where $c$ is the numerical constant, independent on $p$ and $q$.

Following [12, we introduce notation

$$
e^{-4 \pi i \rho}=\frac{\sin 2 \pi(\sigma+\eta)}{\sin 2 \pi \eta} .
$$

Using this and the connection formulae (7), (10), we can re-write the differential form $\frac{1}{4}\left(\alpha d \beta-2 i b_{+} d b_{-}\right)$as the differential form in variables $\eta, \rho, \sigma$ and $\nu$,

$$
\begin{gathered}
\frac{1}{4}\left(\alpha d \beta-2 i b_{+} d b_{-}\right)=-8 \pi i(\sigma d \eta+i \nu d \rho)+2 \pi i d \eta-(12-48 \sigma) \ln 2 d \sigma \\
+(i \pi+4 \ln 2) \nu d \nu+(1-4 \sigma) d \ln \frac{\Gamma(1-2 \sigma)}{\Gamma(2 \sigma)}+2 i \nu d \ln \Gamma(1+i \nu) .
\end{gathered}
$$

Therefore, we can re-write (54) as

$$
\begin{gathered}
\ln \frac{C_{\infty}}{C_{0}}=\nu^{2}+4 \sigma-8 \sigma^{2}-i \nu+2 \pi i \eta-12 \sigma \ln 2+24 \sigma^{2} \ln 2+\frac{i \pi \nu^{2}}{2}+2 \nu^{2} \ln 2 \\
-8 \pi i \int(\sigma d \eta+i \nu d \rho)+\int(1-4 \sigma) d \ln \frac{\Gamma(1-2 \sigma)}{\Gamma(2 \sigma)}+\int 2 i \nu d \ln \Gamma(1+i \nu)+c .
\end{gathered}
$$


It remains to evaluate the integrals in (55). For the integrals involving the $\Gamma$-functions one gets,

$$
\begin{aligned}
& \int(1-4 \sigma) d \ln \frac{\Gamma(1-2 \sigma)}{\Gamma(2 \sigma)}=\ln \frac{\Gamma(1-2 \sigma)}{\Gamma(2 \sigma)}-4 \sigma+8 \sigma^{2}+2 \ln (G(1-2 \sigma) G(1+2 \sigma))+c, \\
& \int 2 i \nu d \ln \Gamma(1+i \nu)=i \nu-\nu^{2}-i \nu \ln (2 \pi)+2 \ln G(1+i \nu)+c,
\end{aligned}
$$

where $G(z)$ is the Barnes G-function and we have used the classical formula,

$$
\int \ln \Gamma(x) d x=\frac{z(1-z)}{2}+\frac{z}{2} \ln (2 \pi)+z \ln \Gamma(z)-\ln G(1+z)+c .
$$

The most challenging, i.e., the first integral in (55) has already been evaluated in [12]. Here is the result.

$$
\int \sigma d \eta+i \nu d \rho=\sigma \eta+i \nu \rho-\mathcal{W}(\sigma, \nu)+c
$$

where the function $\mathcal{W}(\sigma, \nu)$ is expressed in terms of the dilogarithm $L i_{2}(z)$,

$$
8 \pi^{2} \mathcal{W}(\sigma, \nu)=L i_{2}\left(-e^{2 \pi i\left(\sigma+\eta-i \frac{\nu}{2}\right)}\right)+L i_{2}\left(-e^{-2 \pi i\left(\sigma+\eta+i \frac{\nu}{2}\right)}\right)-4 \pi^{2} \eta^{2}+\pi^{2} \nu^{2},
$$

Taking into account yet another classical formula,

$$
L i_{2}\left(e^{2 \pi i z}\right)=-2 \pi i \ln \hat{G}(z)-2 \pi i z \ln \frac{\sin (\pi z)}{\pi}-\pi^{2} z(1-z)+\frac{\pi^{2}}{6},
$$

where

$$
\hat{G}(z)=\frac{G(1+z)}{G(1-z)},
$$

and the elementary relation,

$$
2 \cos \pi\left(\sigma+\eta \pm \frac{i \nu}{2}\right)=e^{i \pi\left( \pm \sigma \mp \eta-\frac{i \nu}{2}-4 \rho\right)}
$$

we arrive at the following final expression for the first integral in (55)

$$
\begin{aligned}
& -8 \pi i \int \sigma d \eta+i \nu d \rho=-8 \pi i \sigma \eta+2 \ln \frac{\hat{G}\left(\sigma+\eta+\frac{1-i \nu}{2}\right)}{\hat{G}\left(\sigma+\eta+\frac{1+i \nu}{2}\right)}-4 \pi \eta^{2}-i \pi \nu^{2}+2 i \ln (2 \pi) \nu \\
& -\frac{3 \pi i \nu^{2}}{2}-2 \pi \sigma^{2}+6 \pi i \eta^{2}+4 \pi i \sigma \eta-2 \pi i \sigma+2 \pi i \eta .
\end{aligned}
$$

Substituting formulae (56), (57), and (61) in 55) we arrive at the equation,

$$
\frac{C_{\infty}}{C_{0}}=c_{1}(2 \pi)^{i \nu} 2^{2 \nu^{2}+\sigma^{2} 24-12 \sigma} e^{2 \pi i\left(\eta^{2}-2 \sigma \eta-\sigma^{2}+2 \eta-\sigma\right)}
$$




$$
\times \frac{\Gamma(1-2 \sigma)}{\Gamma(2 \sigma)}\left(\frac{G(1+i \nu) G(1+2 \sigma) G(1-2 \sigma) \hat{G}\left(\sigma+\eta+\frac{1-i \nu}{2}\right)}{\hat{G}\left(\sigma+\eta+\frac{1+i \nu}{2}\right)}\right)^{2},
$$

where $c_{1}$ is a numerical constant. We know, that if $u=0, \sigma=\eta=\frac{1}{4}, \nu=0$, then $\tau=\operatorname{const} \cdot e^{\frac{x^{2}}{8}}$ and $C_{\infty}=C_{0}$. This choice of parameters satisfies conditions (9). Hence,

$$
c_{1}=\frac{2^{\frac{3}{2}} e^{-i \frac{\pi}{4}}}{\pi\left(G\left(\frac{1}{2}\right)\right)^{4}}
$$

To complete the proof of Theorem 1 we only need now to lift the technical assumption (44). This can be justified by noticing that the both sides of (62) are analytic functions of the Riemann-Hilbert data. (For the left hand side it follows from the general Birkhoff-GrothendieckMalgrange theory.)

Remark 5. The variables $(\eta, \sigma)$ and $(-i \rho, \nu)$ are canonical variables. In fact, one has that [12],

$$
\Omega=32 \pi i d \eta \wedge d \sigma=32 \pi d \rho \wedge d \nu .
$$

The function $\mathcal{W}$ was introduced in [12] as the generating function of the canonical transformation

$$
(\eta, \sigma) \rightarrow(-i \rho, \nu)
$$

Indeed, using (59), (60) and the fact that $L i_{2}^{\prime}(z)=-z^{-1} \ln (z-1)$, one can show that [12],

$$
\eta=\frac{\partial \mathcal{W}}{\partial \sigma}, \quad \text { and } \quad i \rho=\frac{\partial \mathcal{W}}{\partial \nu}
$$

The last equation is also equivalent to the integral formula (58).

In [12], and in fact earlier in the pioneering works [5], [6], the derivation of the constant terms in the asymptotics of the tau-functions was based on the heuristic assumption (followed from the conformal block representation of the tau-functions) that these constants are related to the generating functions of the relevant canonical transformations between the canonical pairs associated with different critical points. In the case of equation (1) the points are 0 and $\infty$ and the generating function is the function $\mathcal{W}$. This is a very important conceptual point, and our analysis justifies it in the case of the Painlevé III equation (1). It is also worth noticing that this hamiltonian interpretation of the ratio $C_{\infty} / C_{0}$ is already present in formula (54). Indeed, this formula tell us that the logarithm of the ratio $C_{\infty} / C_{0}$ is, up to the elementary function, $\nu^{2}+\alpha^{2} / 8-i \nu$, the generating function of the canonical transformation between the Cauchy data $(\alpha, \beta)$ and asymptotic at infinity data $\left(b_{+}, b_{-}\right)$.

\section{Proof of the ILT-Conjecture}

In [12] different $\tau$-function was introduced

$$
\tau_{m}\left(2^{-12} x^{4}\right)=(\tau(x))^{\frac{1}{2}} x^{\frac{1}{4}} e^{\frac{i u(x)}{4}},
$$




$$
\begin{aligned}
& \tau_{m}\left(2^{-12} x^{4}\right)=\frac{2^{-12 \sigma^{2}} x^{4 \sigma^{2}}}{G(1+2 \sigma) G(1-2 \sigma)}(1+o(1)), \quad x \rightarrow 0, \\
& \tau_{m}\left(2^{-12} x^{4}\right)=\chi(\sigma, \nu) e^{\frac{i \pi \nu^{2}}{4}} 2^{\nu^{2}}(2 \pi)^{-\frac{i \nu}{2}} G(1+i \nu) x^{\frac{\nu^{2}}{2}+\frac{1}{4}} e^{\frac{x^{2}}{16}+\nu x}(1+o(1)), \quad x \rightarrow \infty .
\end{aligned}
$$

But from formulae (11), 12), 63) we also have

$$
\begin{aligned}
& \tau_{m}\left(2^{-12} x^{4}\right)=C_{0}^{\frac{1}{2}} e^{-\frac{i \pi}{4}+i \pi \eta} 2^{\frac{3}{2}-6 \sigma} x^{4 \sigma^{2}} \frac{\Gamma(1-2 \sigma)}{\Gamma(2 \sigma)}(1+o(1)), \quad x \rightarrow 0, \\
& \tau_{m}\left(2^{-12} x^{4}\right)=C_{\infty}^{\frac{1}{2}} x^{\frac{\nu^{2}}{2}+\frac{1}{4}} e^{\frac{x^{2}}{16}+\nu x}(1+o(1)), \quad x \rightarrow \infty .
\end{aligned}
$$

From formulae 64), 65), 666), 67) we get

$$
\chi(\sigma, \nu, \eta)=\frac{C_{\infty}^{\frac{1}{2}}}{C_{0}^{\frac{1}{2}}} \frac{(2 \pi)^{\frac{i \nu}{2}} 2^{-\frac{3}{2}-\nu^{2}-12 \sigma^{2}+6 \sigma} e^{-\frac{i \pi \nu^{2}}{2}-i \pi \eta+\frac{i \pi}{4}}}{G(1+i \nu) G(1+2 \sigma) G(1-2 \sigma)}\left(\frac{\Gamma(2 \sigma)}{\Gamma(1-2 \sigma)}\right)^{\frac{1}{2}} .
$$

Substituting here expression for $\frac{C_{\infty}}{C_{0}}$, we get the formula conjectured in [12]

$$
\chi(\sigma, \nu, \eta)=(2 \pi)^{i \nu-\frac{1}{2}} e^{i \pi\left(\eta^{2}-2 \sigma \eta-\sigma^{2}+\eta-\sigma-\frac{\nu^{2}}{4}+\frac{1}{8}\right)} \frac{2^{-\frac{1}{4}}}{G^{2}\left(\frac{1}{2}\right)} \frac{\hat{G}\left(\sigma+\eta+\frac{1-i \nu}{2}\right)}{\hat{G}\left(\sigma+\eta+\frac{1+i \nu}{2}\right)} .
$$

\section{Hamiltonian meaning of Malgrange-Bertola Differen- tial form}

One can rewrite 42

$$
\begin{gathered}
\omega=-\frac{x d x}{4}-\frac{\mathcal{H} d x}{4}-\frac{x \mathcal{H}_{p} d p}{4}-\frac{v u_{p} d p}{4}-\frac{x \mathcal{H}_{q} d q}{4}-\frac{v u_{q} d q}{4} \\
=-\frac{x d x}{4}-\frac{d(x \mathcal{H})}{4}+\frac{x \mathcal{H}_{x} d x}{4}-\frac{v d u}{4}+\frac{v u_{x} d x}{4} .
\end{gathered}
$$

It follows from (1) that

$$
x \mathcal{H}_{x}+v u_{x}=\mathcal{H}
$$

Using this formula we get

$$
\omega=-\frac{1}{4} d\left(\frac{x^{2}}{2}+x \mathcal{H}\right)+\frac{1}{4}(\mathcal{H} d x-v d u) .
$$

We want to emphasize that all the objects are considered as the functions of the triple $(x, p, q)$ and all the differentials are taken with respect to all these three variables.

From (68) it follows that up to the multiplication by -4 and the subtraction of a total differential, the Malgrange-Bertola form $\omega$ coincides with the form

$$
v d u-\mathcal{H} d x \equiv v u_{x} d x+v u_{p} d p+v u_{q} d q-\mathcal{H} d x .
$$


The restriction of this form on a trajectory of the Hamiltonian system (1), i.e. on the curve,

$$
p=\text { const }, \quad q=\text { const }
$$

in the extended space of the monodromy data $\{(x, p, q)\}$ coincides with the form

$$
d S(x)=v u_{x} d x-\mathcal{H} d x
$$

where $S(x)$ is the classical action evaluated on the trajectory $(70)$. Hence, the MalgrangeBertola form $\omega$ can be treated as a natural extension of the canonical form $\mathcal{H} d x-v u_{x} d x$. It follows then, that the tau-function itself can be identified with the classical action. More precisely, along any classical trajectory, we have that

$$
d \ln \tau \equiv \frac{d \ln \tau}{d x} d x=-\frac{1}{4} d S-\frac{1}{4} d(x \mathcal{H}) \equiv\left(-\frac{1}{4} \frac{d S}{d x}-\frac{1}{4} \frac{d(x \mathcal{H})}{d x}\right) d x .
$$

Of course, this differential identity can be easily (after it is written) checked directly. In its turn, it allows us to write the following representation for the ratio $C_{\infty} / C_{0}$ in terms of the regularized action integral,

$$
\ln \frac{C_{\infty}}{C_{0}}=\lim _{t_{0} \rightarrow 0} \lim _{t_{1} \rightarrow+\infty}\left(\int_{t_{0}}^{t_{1}} \frac{\mathcal{H}-v u_{x}}{4} d x-\left.x \mathcal{H}\right|_{t_{0}} ^{t_{1}}-\frac{t_{1}^{2}}{8}-2 \nu t_{1}-\nu^{2} \ln t_{1}-\frac{\alpha^{2}}{8} \ln t_{0}\right) .
$$

It is worth noticing that, unlike the integral $\int \mathcal{H} d x$, the action integral suits well to the differentiation with respect to $p$ and $q$; indeed, after the relevant integration by part the remaining integral term would disappear in view of (1). Therefore, equation $(72)$ provides us with the possibility of an alternative derivation of our key formula (53). This derivation would be very similar to the evaluation of the action integral of the McCoy-Tracy-Wu solution of the PIII equation in [13].

Remark 6. Observe that the extended (with respect to $x, p, q$ ) differential of the form vdu-Hdx is the symplectic form $\Omega$. Therefore, the fact that the Malgrange-Bertola form differs from the form $-\frac{1}{4}(v d u-H d x)$ by a total differential is a fact of general theory; indeed, the (extended) differentials of the both forms coincide - they both are the same 2-form, i.e. $-\frac{1}{4} \Omega$. The additional information we are obtaining in (68) is the explicit evaluation of this total differential. This allows us to relate the tau-function and the action differential explicitly which would be important for the alterantive evaluation of the tau-constant via the action integral.

It might seem quite surprising that one needed to start with the Malgrange-Bertola form in order to discover a rather simple differential identity 71 . The absence of the very idea that the logarithm of the tau-function might differ from the classical action just by a total differential partially explains this. We now believe that the similar fact should be true for any isomonodrtomy tau-function, although it has been apparently missing in the general monodrmy theory of linear systems 1 .

\footnotetext{
${ }^{1}$ In 2000, the first co-author together with Percy Deift tried to use technique of [13] for evolution of the constant factors in the asymptotics of the Painlevé $\mathrm{V}$ tau-function associated with the sine-kernel. We failed then because we did not have the analog of the relation (71) for the Painlevve $\mathrm{V}$ tau - function we were working with. Perhaps, it would make sense to revisit the issue now (although, the relevant constant factors have already been evaluated since then).
} 


\section{Acknowledgements}

The work was supported in part by NSF grant DMS-1361856, and by the SPbGU grant N 11.38.215.2014. The authors are also grateful to M. Bertola for very useful comments.

\section{Appendix. The Derivation of Estimates (46) and (47).}

Substitution of the extended asymptotics 45 into the right hand side of 42 leads to the following expressions for its individual terms.

$$
\begin{aligned}
\cdot \frac{x u_{x}^{2}}{8} & =-\frac{b_{+}^{2} e^{2 i x} x^{2 i \nu}}{8}-\frac{b_{-}^{2} e^{-2 i x} x^{-2 i \nu}}{8}-\frac{b_{+}^{2} e^{2 i x} x^{2 i \nu-1}}{32}\left(6 i \nu^{2}+2 \nu+3 i\right) \\
& +\frac{b_{-}^{2} e^{-2 i x} x^{-2 i \nu-1}}{32}\left(6 i \nu^{2}-2 \nu+3 i\right)-\nu-\frac{\nu^{2}}{x}+\frac{b_{+}^{4} e^{4 i x} x^{4 i \nu-1}}{64}+\frac{b_{-}^{4} e^{-4 i x} x^{-4 i \nu-1}}{64}+O\left(x^{-2+6|\Im \nu|}\right) ;
\end{aligned}
$$

- $-\frac{x}{4}(\cos u-1)=\frac{b_{+}^{2} e^{2 i x} x^{2 i \nu}}{8}+\frac{b_{-}^{2} e^{-2 i x} x^{-2 i \nu}}{8}-\nu-\frac{b_{+}^{4} e^{4 i x} x^{4 i \nu-1}}{64}-\frac{b_{-}^{4} e^{-4 i x} x^{-4 i \nu-1}}{64}$

$$
+\frac{b_{+}^{2} e^{2 i x} x^{2 i \nu-1}}{32}\left(6 i \nu^{2}+2 \nu-i\right)-\frac{b_{-}^{2} e^{-2 i x} x^{-2 i \nu-1}}{32}\left(6 i \nu^{2}-2 \nu-i\right)+O\left(x^{-2+6|\Im \nu|}\right)
$$

- $\frac{x^{2}}{4} u_{p} \sin u=\frac{b_{+} b_{+p} e^{2 i x} x^{2 i \nu}}{16}\left(6 i \nu^{2}+5 \nu-i\right)+\frac{b_{-} b_{+p} x}{4}+\frac{b_{+} b_{+p} e^{2 i x} x^{2 i \nu+1}}{4}-\frac{b_{+}^{3} b_{+p} e^{4 i x} x^{4 i \nu}}{16}$

$$
\begin{gathered}
-\frac{3 b_{-}^{3} b_{+p} e^{-2 i x} x^{-2 i \nu}}{64}+\frac{b_{-} b_{+} \nu}{4}+\frac{i b_{+}^{2} \nu_{p} e^{2 i x} x^{2 i \nu+1} \ln x}{4}-\frac{i b_{-}^{2} \nu_{p} e^{-2 i x} x^{-2 i \nu+1} \ln x}{4} \\
-\frac{i b_{+}^{4} \nu_{p} e^{4 i x} x^{4 i \nu} \ln x}{16}-\frac{b_{-}^{2} \nu_{p} e^{-2 i x} x^{-2 i \nu} \ln x}{16}\left(6 \nu^{2}+2 i \nu-1\right)-\frac{b_{+}^{2} \nu_{p} e^{2 i x} x^{2 i \nu} \ln x}{16}\left(6 \nu^{2}-2 i \nu-1\right) \\
+\frac{b_{-p} b_{+} x}{4}+\frac{b_{-} b_{-p} e^{-2 i x} x^{-2 i \nu+1}}{4}-\frac{3 b_{+}^{3} b_{-p} e^{2 i x} x^{2 i \nu}}{64}-\frac{b_{-}^{3} b_{-p} e^{-4 i x} x^{-4 i \nu}}{16}+\frac{b_{+} b_{-p} \nu}{4} \\
+\frac{i b_{-}^{4} \nu_{p} e^{-4 i x} x^{-4 i \nu} \ln x}{16}-\frac{b_{-} b_{-p} e^{-2 i x} x^{-2 i \nu}}{16}\left(6 i \nu^{2}-5 \nu-i\right)+\frac{b_{+}^{2} \nu_{p} e^{2 i x} x^{2 i \nu}}{8}(3 i \nu-1) \\
-\frac{b_{-}^{2} \nu_{p} e^{-2 i x} x^{-2 i \nu}}{8}(3 i \nu+1)+\nu_{p} \nu+O\left(x^{-1+6|\Im \nu|}\right) ;
\end{gathered}
$$

- $\frac{x^{2}}{4} u_{q} \sin u=\{p \rightarrow q\}$ 
- $\frac{x^{2}}{4} u_{x} u_{p x}=-\frac{b_{+} b_{+p} e^{2 i x} x^{2 i \nu+1}}{4}-\frac{i b_{+}^{2} \nu_{p} e^{2 i x} x^{2 i \nu+1} \ln x}{4}-\frac{b_{-} b_{-p} e^{-2 i x} x^{-2 i \nu+1}}{4}$

$$
\begin{gathered}
+\frac{i b_{-}^{2} \nu_{p} e^{-2 i x} x^{-2 i \nu+1} \ln x}{4}-\frac{b_{+}^{2} \nu_{p} e^{2 i x} x^{2 i \nu}}{8}(3 i \nu+1)-\frac{b_{+} b_{+p} e^{2 i x} x^{2 i \nu}}{16}\left(6 i \nu^{2}+\nu+3 i\right)+\frac{b_{-} b_{+p} x}{4} \\
+\frac{b_{+} b_{-p} x}{4}-\nu_{p} \nu+\frac{b_{+} b_{-p} \nu}{4}+\frac{b_{-} b_{+p} \nu}{4}+\frac{b_{+}^{2} \nu_{p} e^{2 i x} x^{2 i \nu} \ln x}{16}\left(6 \nu^{2}-2 i \nu+3\right)+\frac{b_{+}^{3} b_{+p} e^{4 i x} x^{4 i \nu}}{16} \\
+\frac{i b_{+}^{4} \nu_{p} e^{4 i x} x^{4 i \nu} \ln x}{16}+\frac{b_{-}^{2} \nu_{p} e^{-2 i x} x^{-2 i \nu} \ln x}{16}\left(6 \nu^{2}+2 i \nu+3\right)+\frac{b_{-} b_{-p} e^{-2 i x} x^{-2 i \nu}}{16}\left(6 i \nu^{2}-\nu+3 i\right) \\
+\frac{b_{-}^{2} \nu_{p} e^{-2 i x} x^{-2 i \nu}}{8}(3 i \nu-1)+\frac{b_{-}^{3} b_{-p} e^{-4 i x} x^{-4 i \nu}}{16}-\frac{i b_{-}^{4} \nu_{p} e^{-4 i x} x^{-4 i \nu} \ln x}{16} \\
-\frac{b_{-}^{3} b_{+p} e^{-2 i x} x^{-2 i \nu}}{64}-\frac{b_{+}^{3} b_{-p} e^{2 i x} x^{2 i \nu}}{64}+O\left(x^{-1+6|\Im \nu|}\right) ;
\end{gathered}
$$

- $\frac{x^{2}}{4} u_{x} u_{q x}=\{p \rightarrow q\}$

- $\frac{x u_{x} u_{p}}{4}=\frac{i b_{+} b_{+p} e^{2 i x} x^{2 i \nu}}{4}-\frac{b_{+}^{2} \nu_{p} e^{2 i x} x^{2 i \nu} \ln x}{4}+\frac{i b_{+} b_{-p}}{4}-2 \nu_{p} \nu \ln x-\frac{i b_{-} b_{+p}}{4}$

$$
-\frac{i b_{-} b_{-p} e^{-2 i x} x^{-2 i \nu}}{4}-\frac{b_{-}^{2} \nu_{p} e^{-2 i x} x^{-2 i \nu} \ln x}{4}+O\left(x^{-1+4|\Im \nu|}\right)
$$

- $\frac{x u_{x} u_{q}}{4}=\{p \rightarrow q\}$.

It is quite remarkable that the substitution of these long expressions into the right hand side of 42 yields to the compact formula 46 .

For asymptotics at zero we get the following estimates.

$$
\begin{gathered}
\frac{x u_{x}^{2}}{8}-\frac{x}{4}(\cos u-1)=\frac{\alpha^{2}}{8 x}+O\left(x^{1-|\Im \alpha|}\right), \\
\frac{x^{2} u_{p} \sin u}{4}=O\left(x^{2-|\Im(\alpha)|}\right), \quad \frac{x^{2} u_{q} \sin u}{4}=O\left(x^{2-|\Im(\alpha)|}\right), \\
\frac{x^{2} u_{x} u_{p x}}{x}=\frac{\alpha \alpha_{p}}{4}+O\left(x^{2-|\Im(\alpha)|}\right), \quad \frac{x^{2} u_{x} u_{q x}}{x}=\frac{\alpha \alpha_{q}}{4}+O\left(x^{2-|\Im(\alpha)|}\right), \\
\frac{x u_{x} u_{p}}{4}=\frac{\alpha \alpha_{p} \ln x}{4}+\frac{\alpha \beta_{p}}{4}+O\left(x^{2-|\Im(\alpha)|} \ln x\right), \\
\frac{x u_{x} u_{q}}{4}=\frac{\alpha \alpha_{q} \ln x}{4}+\frac{\alpha \beta_{q}}{4}+O\left(x^{2-|\Im(\alpha)|} \ln x\right) .
\end{gathered}
$$

These equations yield at once (47).

\section{References}

[1] E. Barouch, B.M. McCoy, C.A. Tracy and T.T. Wu, Zero field susceptibility of the twodimensional Ising model near $T_{c}$, Phys. Rev. Lett. 31 (1973) 1409-1411. 
[2] M. Bertola, The dependence on the monodromy data of the isomonodromic tau function, Commun. Math. Phys. 294, 539-579 (2010).

[3] P. Deift, A. Its, I. Krasovsky, On the asymptotic analysis of a Toeplitz determinant with singularities, Random Matrices MSRI Publications, Volume 65, 2014, arXiv: 1206.1292 (2012).

[4] A. S. Fokas, A. R. Its, A. A. Kapaev, V. Yu. Novokshenov, Painlevé transcendents: the Riemann- Hilbert approach, Mathematical Surveys and Monographs 128, AMS, Providence, RI, (2006).

[5] O. Gamayun, N. Iorgov, O. Lisovyy, Conformal field theory of Painlevé VI JHEP 10, (2012), 038; arXiv:1207.0787 [hep-th].

[6] O. Gamayun, N. Iorgov, O. Lisovyy, How instanton combinatorics solves Painlevé VI, V and III's, J. Phys. A46, (2013), 335203; arXiv:1302.1832 [hep-th].

[7] N. Iorgov, O. Lisovyy, Yu. Tykhyy, Painlevé VI connection problem and monodromy of $c=1$ conformal blocks, J. High Energy Phys. 12, (2013), 029; arXiv:1308.4092 [hep-th].

[8] M. Jimbo, Monodromy problem and the boundary condition for some Painlevé equations, Publ. Res. Inst. Math. Sci., 18, N 3, (1982) 1137-1161.

[9] M. Jimbo, T. Miwa, K. Ueno, Monodromy preserving deformation of linear ordinary differential equations with rational coefficients, Physica D 2 (1981) 306-352.

[10] A.R. Its and V.Yu. Novokshenov, The Isomonodromy Deformation Method in the Theory of Painlevé Equations, Lect. Notes in Math., 1191, Springer-Verlag, (1986).

[11] A.V. Kitaev, The method of isomonodromic deformations and the asymptotics of the solutions of the "complete" third Painlevé equation, Mat. Sbornik 134 (176) (1987) no 3, 421-444 (English Transl.: Math. USSR-Sb. 62 (1989) no 2, 421-444).

[12] A. Its, O. Lisovyy, Y. Tykhyy Connection Problem for the Sine-Gordon/Painlevé III TauFunction and Irregular Conformal Blocks, International Mathematics Research Notices, 22 pages, (2014).

[13] S. Lukyanov, A. Zamolodchikov, Exact expectation values of local fields in quantum sineGordon model arXiv: hep-th/9611238, 1996

[14] B. Malgrange, Sur les Déformations Isomonodromiques, I. Singularités Regulieres, in Mathematics and Physics (Paris, 1979/1982), 37 of Prog. Math., pages 401-426 (1983), Birkhäuser Boston, Boston, MA.

[15] B.M. McCoy, C.A. Tracy and T.T. Wu, Painlevé functions of the third kind, J. Math. Phys. 18 (1977) No 5, 1058-1092.

[16] J. M. Myers, Wave scattering and the geometry of a strip, J. Math. Phys. 6 (1965), 1839-1846.

[17] D. G. Niles, The Riemann-Hilbert-Birkhoff inverse monodromy problem and connection formulae for the third Painlevé transcendents, PhD Thesis, Purdue University, (2009) 
[18] V. Yu. Novokshenov, On the asymptotics of the general real solution of the Painlevé equation of the third kind, Sov. Phys. Dokl. 30, (1985), 666 - 668.

[19] K. Okamoto, On the $\tau$-function of the Painlevé equations, Physica D, 2 (1981) No. 3, 525-535.

[20] C. A. Tracy, Asymptotics of the $\tau$-function arising in the two-dimensional Ising model, Comm. Math. Phys. 142, (1991), 297-311. 